\title{
Lateral force calibration in atomic force microscopy: A new lateral force calibration method and general guidelines for optimization
}

\author{
Rachel J. Cannara \\ Physics Department, University of Wisconsin-Madison, 1150 University Ave., Madison, Wisconsin 53706 \\ Michael Eglin ${ }^{\text {a) }}$ and Robert W. Carpick ${ }^{\text {b) }}$ \\ Engineering Physics Department, University of Wisconsin-Madison, 1500 Engineering Dr., Madison, \\ Wisconsin 53706
}

(Received 30 January 2006; accepted 20 March 2006; published online 5 May 2006; publisher error corrected 22 May 2006)

\begin{abstract}
Proper force calibration is a critical step in atomic and lateral force microscopies (AFM/LFM). The recently published torsional Sader method [C. P. Green et al., Rev. Sci. Instrum. 75, 1988 (2004)] facilitates the calculation of torsional spring constants of rectangular AFM cantilevers by eliminating the need to obtain information or make assumptions regarding the cantilever's material properties and thickness, both of which are difficult to measure. Complete force calibration of the lateral signal in LFM requires measurement of the lateral signal deflection sensitivity as well. In this article, we introduce a complete lateral force calibration procedure that employs the torsional Sader method and does not require making contact between the tip and any sample. In this method, a colloidal sphere is attached to a "test" cantilever of the same width, but different length and material as the "target" cantilever of interest. The lateral signal sensitivity is calibrated by loading the colloidal sphere laterally against a vertical sidewall. The signal sensitivity for the target cantilever is then corrected for the tip length, total signal strength, and in-plane bending of the cantilevers. We discuss the advantages and disadvantages of this approach in comparison with the other established lateral force calibration techniques, and make a direct comparison with the "wedge" calibration method. The methods agree to within 5\%. The propagation of errors is explicitly considered for both methods and the sources of disagreement discussed. Finally, we show that the lateral signal sensitivity is substantially reduced when the laser spot is not centered on the detector. (C) 2006 American Institute of Physics. [DOI: 10.1063/1.2198768]
\end{abstract}

\section{INTRODUCTION}

Atomic and lateral force microscopies (AFM/LFM) are well established and popular techniques not only for imaging small-scale surface morphology but also as important tools for studying interfacial forces with pico-Newton-scale force resolution. $^{1,2}$ As a result, there are several established approaches to force calibration. To achieve consistent results between different laboratories, it is important to implement the most accurate calibration method(s) that are practical for a given experiment, and to verify their reliability. Here, we present a new procedure that simplifies lateral force calibration in AFM, and we address the key aspects of lateral force calibration that are important for reliability.

Figure 1 shows the arrangement of the cantilever, laser, and position-sensitive detector (PSD) in the popular opticalbeam-deflection LFM. $^{3-5}$ A focused laser beam reflects off the back of the cantilever and onto the PSD, which consists of four photosensitive sectors (or quadrants) which we label $A 1, A 2, B 1$, and $B 2$. Normal or lateral forces applied to the

\footnotetext{
${ }^{a}$ Now at Blaser Swisslube AG, Winterseistrasse, CH-3415 Hasle-Ruegsau, Switzerland.

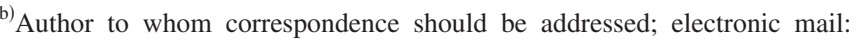
carpick@engr.wisc.edu
}

tip cause the cantilever to bend or twist, respectively. These forces change the angles of reflection of the laser, causing the laser spot on the PSD to displace in the vertical or horizontal direction, respectively. The PSD separately measures the normal and lateral signals, $V_{\text {norm }}=\left(V_{A 1}+V_{A 2}\right)-\left(V_{B 1}+V_{B 2}\right)$ and $V_{\text {lat }}=\left(V_{A 1}+V_{B 1}\right)-\left(V_{A 2}+V_{B 2}\right)$, that vary in proportion to the bending and twisting of the cantilever. To convert these voltage signals into units of force, one needs the lateral and normal force calibration factors, $\alpha$ and $\beta$, respectively, where the corresponding forces, $F_{\text {lat }}$ and $F_{\text {norm }}$, are given by

$$
F_{\text {lat }}=\alpha \times \Delta V_{\text {lat }}
$$

and

$$
F_{\text {norm }}=\beta \times \Delta V_{\text {norm }} .
$$

Each $\Delta V$ represents the change in the respective signal due to an applied force in the respective direction relative to any offset (nonzero voltage) of the signal that is present when no force is applied.

Normal force calibration is straightforward. The first step is to measure the normal deflection sensitivity, $s_{\text {norm }}$, in units of normal signal Volts per vertical displacement of the tip end of the cantilever. The deflection sensitivity depends on the laser intensity, PSD sensitivity and gain, and the geometry of the optical setup, including the cantilever length 


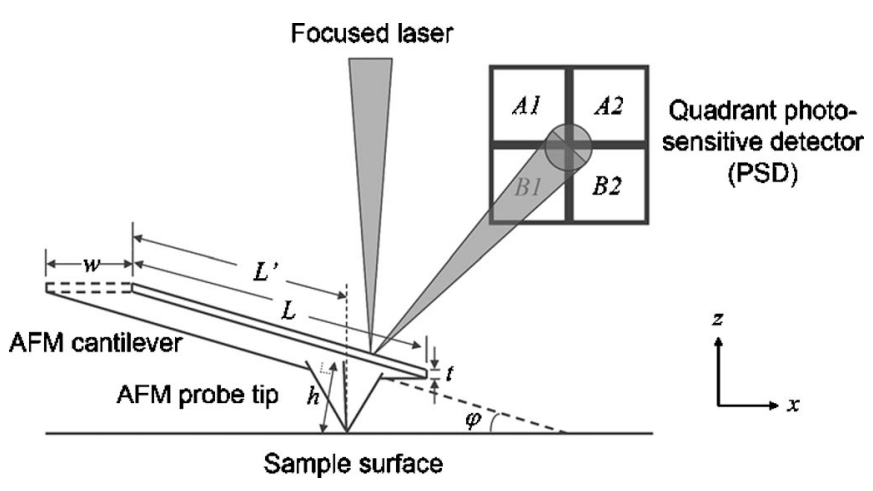

FIG. 1. The optical arrangement in an atomic force microscope. A focused laser beam is reflected off the back of the cantilever onto a four-quadrant photosensitive detector (PSD). The amount by which the cantilever bends and twists in response to normal and lateral forces corresponds to variations in the top minus bottom and left minus right signals, respectively. $L$ and $w$ are the length and width of the cantilever, respectively; $L^{\prime}$ is the distance from the fixed end of the cantilever to the position near the cantilever's free end where the tip is attached or integrated into the cantilever beam; and $h$ is the distance from the tip apex to the neutral axis of the cantilever (the tip height plus half the thickness of the cantilever).

and the distance between the cantilever and the detector. The normal deflection sensitivity is easily obtained by measuring the slope of a normal signal versus vertical piezo displacement plot on a stiff, hard surface. A key point here is that the vertical displacement of the tip is assumed to be equal to the imposed vertical displacement of the piezo. This will not be accurate if the sample is sufficiently compliant to accommodate a significant portion of the imposed vertical displacement through contact deformation. As well, even in the case of a stiff, hard sample, this measurement is only as accurate as the $z$ calibration of the piezo.

The second step is to determine the normal spring constant, $k_{\text {norm }}^{\prime}$, measured at the tip or colloidal sphere position. Once this is known, the complete normal force calibration factor can be determined as,

$$
\beta=\frac{k_{\text {norm }}^{\prime}}{s_{\text {norm }} \cos \varphi},
$$

where $\varphi$ is the angle of the cantilever with respect to the sample surface. (Note that this angle may differ from the tilt angle of the cantilever relative to the AFM $x-y$ scan plane, e.g., the angle for which it may be necessary to compensate in a variable-load experiment. ${ }^{6}$ ) In the case of a rectangular cantilever, the normal spring constant is given by

$$
k_{\text {norm }}^{\prime}=\frac{E t^{3} w}{4 L^{\prime 3}},
$$

where $E, t$, and $w$ are its Young's modulus, thickness, and width, respectively. $L^{\prime}$ is the distance from the base of the cantilever to the tip position, as opposed to the full length, $L$, of the cantilever (Fig. 1).

The flexural Sader method ${ }^{7}$ yields the normal spring constant, $k_{\text {norm }}$, at the end of the cantilever based on its hydrodynamic damping in a fluid medium (e.g., in air). ${ }^{8}$ One does not need to know the thickness of the cantilever or its Young's modulus. $k_{\text {norm }}$ is determined by measuring the cantilever's planar dimensions (length and width) and its resonance frequency and quality factor in air (or any other suit- able fluid medium). ${ }^{9}$ The Sader method improves the accuracy of normal force calibration in comparison with the nominal values provided by the manufacturer. There are several other methods available for normal force calibration, ${ }^{10-12}$ but the Sader method has emerged as the most convenient and accurate.

The normal spring constant, $k_{\text {norm }}^{\prime}$, is related to $k_{\text {norm }}$ by

$$
k_{\text {norm }}^{\prime}=k_{\text {norm }}\left(\frac{L}{L^{\prime}}\right)^{3} \text {. }
$$

This adjustment for $L^{\prime}$ accounts for offset of the tip or colloidal sphere from the free end of the cantilever, and this correction is non-negligible for many popular commercial cantilevers because of the strong (cubic) dependence of the maximum deflection of a cantilever beam on the distance between the base of the cantilever and the position at which load is applied, as defined in Eq. (4). ${ }^{13}$

The lateral force calibration is more challenging. One key reason for this is that the lateral deflection sensitivity is more difficult to determine than the normal deflection sensitivity for conventional cantilevers for the following reason. The lateral stiffness of AFM cantilevers is typically much higher than the normal stiffness, and this makes it more difficult to measure. For example, the torsional spring constant, $\kappa^{\prime}$, for a rectangular cantilever, measured at the tip or colloidal sphere, is given by

$$
\kappa^{\prime}=\frac{G t^{3} w}{3 L^{\prime}},
$$

where $G$ is the shear modulus of the cantilever. The lateral spring constant, $k_{\text {lat }}^{\prime}$, measured at the same position is related to $\kappa^{\prime}$ as follows:

$$
k_{\text {lat }}^{\prime}=\frac{\kappa^{\prime}}{h^{2}},
$$

where $h$ is the torsional moment arm, i.e., the distance from the line of action of the lateral force (which acts on the end of the tip) to the twisting axis of the cantilever (Fig. 1), i.e., colloid radius or tip height plus the distance to the neutral axis of the cantilever. The ratio of the normal to the lateral spring constant is therefore

$$
\frac{k_{\text {norm }}^{\prime}}{k_{\text {lat }}^{\prime}}=\frac{3 E}{4 G}\left(\frac{h}{L^{\prime}}\right)^{2},
$$

where $E$ is always greater than $G,{ }^{14}$ but $h$ is much less than $L^{\prime}$, so that $\left(h / L^{\prime}\right)^{2}$ is very small. Consequently, $k_{\text {norm }}^{\prime} / k_{\text {lat }}^{\prime}$ can be as low as 0.001 for a rectangular cantilever.

The total lateral stiffness of the cantilever-tip-contact system, $k_{\text {lat,tot, }}$ is obtained from a sum of inverses of the lateral force constant of the cantilever, $k_{\text {lat }}^{\prime}$, measured at the sphere or tip position, the lateral stiffness of the tip or sphere itself, $k_{\text {lat,tip }}$, and the lateral stiffness of the contact, $k_{\text {lat,cont, }}$

$$
k_{\text {lat,tot }}^{-1}=k_{\text {lat }}^{-1}+k_{\text {lat,tip }}^{-1}+k_{\text {lat,cont }}^{-1} \text {. }
$$

Consequently, for a typical integrated AFM tip and sample surface, the lateral contact stiffness, which is often comparable to or less than the lateral stiffness of the cantilever ${ }^{15}$ and tip, ${ }^{16}$ significantly reduces the slope of the lateral force versus lateral displacement plot. In other words, this slope, 
TABLE I. Lateral force calibration methods, their corresponding references, key assumptions and measured variables, and required materials/probes.

\begin{tabular}{|c|c|c|c|c|}
\hline $\begin{array}{l}\text { Lateral } \\
\text { force } \\
\text { calibration } \\
\text { method }\end{array}$ & $\begin{array}{l}\text { Relevant } \\
\text { references }\end{array}$ & Key assumption(s) & $\begin{array}{l}\text { Key measured } \\
\text { variable(s) }\end{array}$ & $\begin{array}{l}\text { Required } \\
\text { materials/probes }\end{array}$ \\
\hline Test probe & This article and 26 & $\begin{array}{l}\text { Same laser intensity } \\
\text { distribution for test } \\
\text { cantilever and cantilever } \\
\text { with integrated tip; } \\
\text { accurate lateral piezo calibration. }\end{array}$ & $\begin{array}{l}\text { Colloid moment arm } \\
\text { and lateral deflection } \\
\text { sensitivity; torsional } \\
\text { spring constant; tip } \\
\text { moment arm. }\end{array}$ & $\begin{array}{l}\text { Colloidal test probe, } \\
\text { GaAs crystal, } \\
\text { cantilever with } \\
\text { integrated tip (to be } \\
\text { calibrated). }\end{array}$ \\
\hline Wedge & 24 and 25 & $\begin{array}{l}\text { Slopes of facets or wedge } \\
\text { surfaces with known } \\
\text { relative tilt angle; } \\
\text { accurate lateral piezo calibration. }\end{array}$ & $\begin{array}{l}\text { Half-width and lateral } \\
\text { offset of friction loop } \\
\text { vs. load; normal } \\
\text { spring constant. }\end{array}$ & $\begin{array}{l}\text { Wedge calibration } \\
\text { grating, cantilever } \\
\text { with colloid, or integrated tip } \\
\text { (to be calibrated). }\end{array}$ \\
\hline $\begin{array}{l}\text { Vertical } \\
\text { lever }\end{array}$ & 20 & $\begin{array}{l}\text { Known loading position } \\
\text { of colloidal probe on } \\
\text { vertical test cantilever. }\end{array}$ & $\begin{array}{l}\text { Lateral deflection } \\
\text { sensitivity; test } \\
\text { cantilever normal } \\
\text { spring constant. }\end{array}$ & $\begin{array}{l}\text { Colloidal probe, rigid } \\
\text { vertical surface, precalibrated } \\
\text { test cantilever. }\end{array}$ \\
\hline $\begin{array}{c}\text { Optical } \\
\text { geometry }\end{array}$ & 19 & $\begin{array}{l}\text { Torsional spring constant } \\
\text { calculated from estimated } \\
\text { modulus and cantilever } \\
\text { dimensions (this can now } \\
\text { be measured } \\
\text { experimentally using the } \\
\text { Sader method). }{ }^{26}\end{array}$ & $\begin{array}{l}\text { PSD dimensions, } \\
\text { distance from cantilever to PSD, } \\
\text { probe moment arm. }\end{array}$ & $\begin{array}{l}\text { Any cantilever-probe } \\
\text { combination. }\end{array}$ \\
\hline $\begin{array}{l}\text { Static } \\
\text { friction }\end{array}$ & 17 and 30 & $\begin{array}{l}\text { Negligible contact } \\
\text { stiffness, accurate lateral } \\
\text { piezo calibration; spring } \\
\text { constant from finite } \\
\text { element model. }\end{array}$ & $\begin{array}{l}\text { Slope of lateral signal } \\
\text { vs. lateral piezo displacement; } \\
\text { lateral spring constant. }\end{array}$ & $\begin{array}{l}\text { Colloidal probe, flat } \\
\text { hard surface. }\end{array}$ \\
\hline
\end{tabular}

which can be observed quite easily at the beginning or "sticking" portion of a friction loop, is emphatically not equal to the lateral deflection sensitivity. An exception to this case usually occurs for micrometer-scale colloidal spheres for which the large contact area produces a large contact stiffness. ${ }^{17}$ In addition, $k_{\text {lat,tip }}$ is typically very large for a colloidal sphere, and may be neglected in this case, as well.

While the normal deflection sensitivity is measured easily from vertical force versus displacement plots on a stiff surface, it is generally more difficult to achieve an equivalent configuration for the lateral case because of the higher lateral stiffness of standard cantilevers. Also, it is necessary to consider any reduction in lateral deflection sensitivity due to lateral in-plane bending that may occur in addition to the torsional response. As reported by Sader and Green, ${ }^{18}$ lateral in-plane bending may be ignored for rectangular cantilevers that satisfy the criterion

$$
\varepsilon \equiv \frac{k_{\text {lat }}^{\prime}}{k_{\text {in-plane }}^{\prime}}=\frac{2}{3(1+\nu)}\left(\frac{t}{h}\right)^{2}\left(\frac{L^{\prime}}{w}\right)^{2} \ll 1,
$$

where $k_{\text {in-plane }}^{\prime}$ is the lateral in-plane bending spring constant measured at the tip or sphere position, and $\nu$ is Poisson's ratio. Since the quotient $1 / 2(1+\nu)=G / E$, Eq. (8) may be rearranged to yield the ratio $G / E$, and the criterion in Eq. (10) may be rewritten in the following way, independent of Poisson's ratio:

$$
\varepsilon=\frac{k_{\text {lat }}^{\prime}}{k_{\text {norm }}^{\prime}}\left(\frac{t}{w}\right)^{2} \ll 1,
$$

or, alternately,

$$
\varepsilon=\frac{\kappa}{k_{\text {norm }}}\left(\frac{t}{h w}\right)^{2}\left(\frac{L^{\prime}}{L}\right)^{2} \ll 1,
$$

where the full spring constants, $\kappa$ and $k_{\text {norm }}$, may be determined by Sader's methods. If Eq. (10), (11), or (12) is not satisfied, lateral in-plane bending will act to reduce the lateral deflection sensitivity. This effect can be significant, as discussed below.

Several lateral force calibration methods work around the challenge of measuring the lateral sensitivity (Table I). The optical geometry method of Liu et al., ${ }^{19}$ static friction method of Cain et al., ${ }^{17}$ and vertical lever method of Ecke et $a l^{20}$ each describe lateral force calibration procedures that are analogous to the normal force calibration described above. Here, we briefly review the established methods.

In the first set of methods, the lateral force calibration factor, $\alpha$, is obtained from the lateral spring constant, $k_{\text {lat }}$, and lateral deflection sensitivity, $s_{\text {lat }}$, such that

$$
\alpha=\frac{k_{\text {lat }}^{\prime}}{s_{\text {lat }}} .
$$

Just as $s_{\text {norm }}$ is the change in normal signal Volts per normal deflection, $s_{\text {lat }}$ is the change in lateral signal Volts for a given lateral displacement of the tip.

In their optical geometry approach, Liu et al. calculate the torsional spring constant based on assumed elastic (shear modulus) and measured or assumed geometric (length, width, thickness, and tip height) properties of the cantilever. They determine the lateral deflection sensitivity by moving the PSD a known amount in the lateral direction (along $x$ in 
Fig. 1), which is converted to an equivalent lateral tip displacement by using measured or assumed values for the tip height and the PSD-cantilever distance.

For their static friction method, Cain et al. calculate the torsional spring constant based on finite element analysis, ${ }^{21}$ but they obtain the lateral deflection sensitivity from the static portion of a friction loop between a colloidal probe and a hard, flat surface. Due to the large sphere diameter, the resulting contact stiffness is much greater than the cantilever stiffness, the effect of the contact stiffness is negligible [Eq. (9)], and an accurate value for the lateral deflection sensitivity is obtained.

Ecke et al. take a different approach in their vertical lever method, obtaining deflection sensitivities for a colloidal probe by pushing the equator of the sphere laterally against a rigid, vertical surface. Then, they push the probe in the lateral direction against a vertically oriented cantilever with a calibrated normal spring constant. This provides both the lateral deflection sensitivity and the lateral force constant.

In addition, the more recent friction force calibration method by Stiernstedt et al. measures friction as an eccentric axial force (i.e., parallel to the long axis of the cantilever) and therefore uses the normal spring constant and vertical deflection sensitivity for calibrating both normal and frictional forces. ${ }^{22}$ The novel method by Choi combines the moment balance equations for a cantilever with information from the lateral signal on a step grating to produce a calibration to separate the cantilever properties from the instrumental properties. ${ }^{23}$

In contrast to these methods, the wedge method, developed by Ogletree $e$ t $a .^{24}$ and extended to colloidal probes by Varenberg et al., ${ }^{25}$ is a scheme for lateral force calibration that bypasses the separate measurement of the lateral force constant and lateral deflection sensitivity altogether. Instead, the ratio of the normal and lateral calibration factors, $S$, is determined, where

$$
\alpha=S \times \beta .
$$

Thus, once $\beta$ is known [e.g., via Sader's method and Eqs. (3) and (5)], then $\alpha$ can be determined. To obtain $\alpha / \beta$, measurements are performed by scanning the AFM tip over a wedgeshaped sample of known geometry. When a surface is tilted locally along the scanning direction, there are geometrically determined components of the normal and frictional forces between the tip and sample that will couple into the lateral and normal force channels. $S$ is determined by comparing the expected normal-to-lateral force ratio to the measured normal-to-lateral signal ratio.

Here, we demonstrate the new test probe calibration procedure, which combines the torsional Sader method ${ }^{26}$ (for experimentally determining torsional spring constants) with a method for measuring the lateral detector sensitivity of the AFM, giving a full calibration from Volts to Newtons for colloidal and integrated tips alike. The method is similar to the vertical lever method, but uses fewer assumptions and a simpler experimental setup, and contact between the tip and any calibration sample is avoided, which is advantageous for tips with sensitive end structure or functionalities.

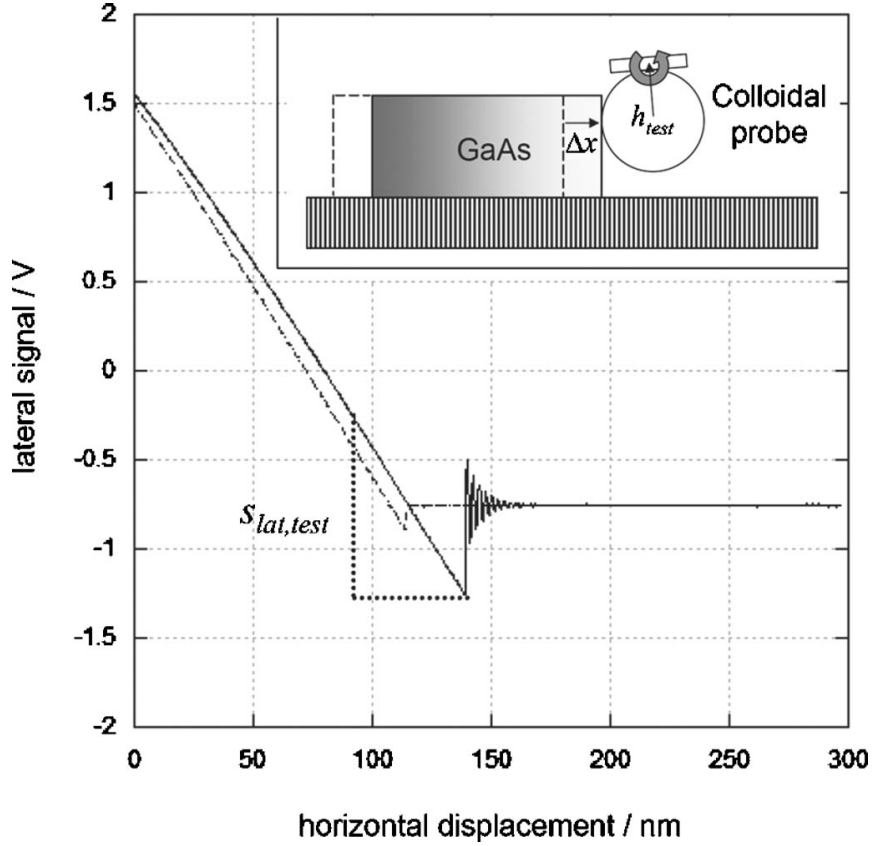

FIG. 2. Lateral force vs. displacement plot. The inset shows the arrangement of the colloidal probe and GaAs sample in the test probe method. When the sample moves horizontally by $\Delta x$, it pushes against the equator of the sphere, causing the cantilever to twist and producing the plot shown. The slope, $s_{\text {lat,test }}$, of this plot is the lateral deflection sensitivity for the test probe. In this example, approach (dashed) and retract (solid) lateral force versus displacement plots taken at a rate of $250 \mathrm{~ms}$ per plot are shown for a displacement range of $300 \mathrm{~nm}$ (i.e., $300 \mathrm{~nm}$ per $250 \mathrm{~ms}$ ).

\section{RESULTS}

\section{A. Test probe method for lateral force calibration}

In the new test probe method, a colloidal sphere is glued to the tip end of a "test" cantilever of the same width and gold coating as the cantilever to be used for experiments (the "target" cantilever). Lateral force versus displacement measurements (at the equator of the sphere) are performed against the flat side of a freshly cleaved gallium arsenide sample, which cleaves at a perfect $90^{\circ}$ angle to the top (100) plane (Fig. 2 inset). The slope of the contact region in the resulting force plot gives the lateral deflection sensitivity, $s_{\text {lat,test }}$. The lateral deflection sensitivity obtained in this way depends on the geometry of the laser beam path, the torsional moment arm, and the total signal on the PSD, but it is independent of the width of the cantilever, as long as the entire laser spot reflected off the cantilever.

Dividing the lateral force constant of the target cantilever by the lateral deflection sensitivity measured for the test probe according to Eq. (13) is not sufficient for calibrating lateral forces; one must also account for changes in the torsional moment arm length and the total signal on the photodiode between the test and the target probes. In addition, test cantilevers should be chosen so that the lateral in-plane bending is negligible (i.e., $\varepsilon_{\text {test }} \ll 1$ ), since it reduces the lateral deflection sensitivity, or in-plane bending of the test cantilever should be identical to the target (i.e., $\varepsilon_{\text {test }}=\varepsilon_{\text {target }}$ ). In general, it is necessary to correct for in-plane bending of the target probe. With torsional moment arm lengths, $h_{\text {target }}$ and $h_{\text {test }}$, and total signals, $T_{\text {target }}$ and $T_{\text {test }}$, for the colloidal test 
probe and new probe, respectively, the lateral deflection sensitivity of the target probe will be

$$
s_{\text {lat, target }}=s_{\text {lat, test }} \frac{\left(T_{\text {target }} / T_{\text {test }}\right)}{\left(h_{\text {target }} / h_{\text {test }}\right)} \frac{\left(1+\varepsilon_{\text {test }}\right)}{\left(1+\varepsilon_{\text {target }}\right)},
$$

where the final term accounts for lateral in-plane bending of the target cantilever relative to the test cantilever.

Equation (15) assumes that the calibration is independent of the laser spot position along the length of the cantilever. This is valid if the laser spot reflects off the back of both the test and target cantilevers at points between the tip or colloidal sphere's location and the free ends of the cantilevers. This is a reasonable assumption, because, for a given applied lateral force, the angle of twist does not change along this portion of the cantilever. This is easily accomplished by always positioning the laser as close to the free end of the cantilever as possible. If the laser spot for one or both cantilevers instead lies between the tip or colloidal sphere's location and the fixed end of the cantilever, then a linear correction is required to account for this offset.

The use of the ratio of total signals also assumes that the spatial distribution of reflected laser intensity on the PSD is sufficiently similar for both cantilevers, even if the total intensity is different. This assumption may break down if one switches to a cantilever of significantly different width or surface coating. To verify that Eq. (15) holds in the case of cantilevers of identical width and reflective coating, we compared the normal deflection sensitivities of the two probes in this work, which had the same width and a gold coating, but came from different wafers. In this case, the relevant moment arms are the lengths, $L_{\text {target }}^{\prime}$ and $L_{\text {test }}^{\prime}$, for the target and test cantilevers, respectively. As a result we can compare the normal deflection sensitivities for the colloidal test probe and target probe in the following way:

$$
s_{\text {norm,target }}=s_{\text {norm,test }} \frac{\left(T_{\text {target }} / T_{\text {test }}\right)}{\left(L_{\text {target }}^{\prime} / L_{\text {test }}^{\prime}\right)} .
$$

We verified this relation from the normal force versus displacement plots taken on a flat Si surface using several different probes. As an example, using digital instruments (DI) Nanoscope IV AFM, we compared the deflection sensitivities for two 40- $\mu \mathrm{m}$-wide, gold-coated cantilevers (with silicon and silicon nitride substrates for the target and test probes, respectively), one with an integrated tip and one with a colloidal sphere (the test probe). In this case, the length of the first cantilever was $L_{\text {target }}^{\prime}=335 \mu \mathrm{m}$, and the length of the test cantilever was $L_{\text {test }}^{\prime}=195 \mu \mathrm{m}$. The normal signal deflection sensitivities were $s_{\text {norm,target }}=8.7 \mathrm{mV} / \mathrm{nm}$ and $s_{\text {norm,test }}$ $=15.3 \mathrm{mV} / \mathrm{nm}$, and the total signal intensities were $T_{\text {target }}$ $=7.1 \mathrm{~V}$ and $T_{\text {test }}=7.2 \mathrm{~V}$. From this, both sides of Eq. (16) agree to within $1 \%$. This shows that the laser spot intensity distribution does not change significantly for distinct cantilevers of the same width and reflective coating, even if they come from different wafers or have different substrate materials.

In the next calibration step, $k_{\text {lat }}^{\prime}$ is calculated from $\kappa$, the torsional spring constant for the full length of the cantilever,

$$
k_{\mathrm{lat}}^{\prime}=\frac{\kappa}{h^{2}}\left(\frac{L}{L^{\prime}}\right),
$$

where $\kappa$ is obtained experimentally via the torsional Sader method. ${ }^{26}$ The resonance frequency and quality factor in air for the first torsional oscillation mode of the target cantilever are measured separately or in situ. The factor $L / L^{\prime}$ accounts for the fact that forces in an experiment are applied at the tip position, not at the end of the cantilever. It is a linear dependence (in contrast to the cubic dependence for the normal force constant), because the angle of twist is linear with cantilever length. The static friction and optical geometry methods may be modified to use the torsional Sader method as well.

We used a UHV350 AFM from RHK Technology (Troy, Michigan) for the lateral calibration test. This instrument has inertial motors that enable the user to make both coarse and fine adjustments to the physical offset of the PSD in both the vertical and horizontal directions. Normal and lateral signals may be decoupled electronically, though we do not employ this function for this calibration.

Deflection sensitivity measurements with the colloidal probe were performed under a controlled environment (nitrogen flushed and pumped to 10 Torr). Lateral force versus displacement plots were obtained by pushing a $70-\mu \mathrm{m}$-diam colloidal glass sphere (attached to a silicon nitride cantilever) against the flat cleaved ( $\overline{1} 10)$ side of a GaAs crystal, using a $300 \mathrm{~nm}$ lateral ramp size in the $\pm x$ direction (perpendicular to the long axis of the cantilever). Any sample with a flat, stiff vertical surface may be used for this calibration. The test probe satisfied Eq. (10), with $\varepsilon_{\text {test }} \cong 0.01$, because the cantilever length $(\sim 200 \mu \mathrm{m})$ is relatively short, and its thickness $[\sim 1 \mu \mathrm{m}$ by scanning electron microscopy (SEM)] is much less than the moment arm and cantilever width. As a result, in-plane bending is negligible, and the deflection sensitivity measured with this probe should accurately describe the lateral sensitivity of the detector.

Figure 2 shows a representative lateral force versus displacement plot and the probe-sample arrangement (Fig. 2 inset). This plot resembles a typical normal force versus displacement plot, including the effect of snap-in and pull-off due to adhesion, which in this case occurs between the sides of the colloid and the GaAs sidewall. For retracting force plots, we measured the lateral deflection sensitivity from the slope between the point at which the positive load is equal in magnitude to the pull-off force and the point at which the probe pulls off. For approaching plots, we do the same except that the snap-in force is the reference point. With the laser spot centered both horizontally and vertically on the PSD, the lateral deflection sensitivity is $2.19 \pm 0.01 \mathrm{mV} / \mathrm{nm}$.

To assess this first part of the test probe method further, we compared it with the static friction method, using the DI AFM. For the same $70 \mu \mathrm{m}$ colloidal probe at zero lateral PSD offset, the two approaches yield similar results (correcting for the doubled length of the cantilever arm with the static friction method): $\sim 64$ and $\sim 68 \mathrm{mV} / \mathrm{nm}$ for the test probe and static friction methods, respectively. Though the result is similar for these two methods, the test probe method improves upon the static friction approach, because the side 
TABLE II. Measured and calculated values and their percent uncertainties.

\begin{tabular}{|c|c|c|c|}
\hline Variable & Parameter (measured) & Value & $\%$ Uncertainty \\
\hline$L$ & Full target cantilever length & $342.8 \pm 0.6 \mu \mathrm{m}$ & 0.18 \\
\hline$L^{\prime}$ & Target probe base-to-tip distance & $332.9 \pm 0.4 \mu \mathrm{m}$ & 0.12 \\
\hline$w$ & Target cantilever width & $38.1 \pm 0.4 \mu \mathrm{m}$ & 1.1 \\
\hline$h_{\text {target }}$ & Target torsional moment arm & $22.34 \pm 0.17 \mu \mathrm{m}$ & 0.76 \\
\hline$h_{\text {test }}$ & Colloid test probe torsional moment arm & $36.05 \pm 0.32 \mu \mathrm{m}$ & 0.89 \\
\hline$f_{\text {norm }}$ & Target normal resonance frequency & $10.5 \pm 0.1 \mathrm{kHz}$ & 0.95 \\
\hline$f_{\text {tors }}$ & Target torsional resonance frequency & $163.3 \pm 0.1 \mathrm{kHz}$ & 0.06 \\
\hline$Q_{\text {norm }}$ & Normal quality factor in air & $49.0 \pm 0.7$ & 1.4 \\
\hline \multirow[t]{2}{*}{$Q_{\text {tors }}$} & Torsional quality factor in air & $204.4 \pm 10.5$ & 5.1 \\
\hline & Parameter (calculated from measurements) & & \\
\hline$S$ & Target wedge calibration sensitivity & $33.7 \pm 1.0$ & 3.0 \\
\hline$s_{\text {lat,test }}$ & Colloid test lateral deflection sensitivity & $2.19 \pm 0.01 \mathrm{mV} / \mathrm{nm}$ & 0.5 \\
\hline$s_{\text {norm,target }}$ & Target normal deflection sensitivity & $1.27 \pm 0.01 \mathrm{mV} / \mathrm{nm}$ & 0.53 \\
\hline$k_{\text {norm,target }}$ & Target full normal spring constant & $0.074 \pm 0.002 \mathrm{~N} / \mathrm{m}$ & 2.7 \\
\hline$k_{\text {norm,target }}^{\prime}$ & Target normal spring constant & $0.080 \pm 0.002 \mathrm{~N} / \mathrm{m}$ & 2.5 \\
\hline$\kappa_{\text {target }}$ & Target torsional spring constant & $2.91 \pm 0.19 \mathrm{nN} \mathrm{m}$ & 6.5 \\
\hline$k_{\text {lat,target }}$ & Target lateral force constant & $6.00 \pm 0.41 \mathrm{~N} / \mathrm{m}$ & 6.8 \\
\hline \multirow[t]{2}{*}{$\beta$} & Target normal force sensitivity factor & $63.3 \pm 1.8 \mathrm{nN} / \mathrm{V}$ & 2.9 \\
\hline & Final results & & \\
\hline$\alpha_{\text {target }}$ & Colloid lateral calibration factor & $1.72 \pm 0.12 \mathrm{nN} / \mathrm{mV}$ & 6.8 \\
\hline$\alpha_{\text {target }}$ & The same, including in-plane bending & $2.39 \pm 0.18 \mathrm{nN} / \mathrm{mV}$ & 7.5 \\
\hline$\alpha_{\text {wedge }}$ & Target wedge lateral calibration factor & $2.25 \pm 0.09 \mathrm{nN} / \mathrm{mV}$ & 4.2 \\
\hline
\end{tabular}

of the colloid instead of its apex touches the calibration sample. Therefore, the apex of the sphere is not subjected to wear or chemical modification during the calibration procedure - an important feature if the colloidal probe is also the target probe in the LFM experiment. We note that lateral in-plane bending may be ignored when the target probe is also the test probe. However, this is not the case in general.

After obtaining the lateral deflection sensitivity with the test probe, we then calculate the lateral force calibration factor, $\alpha_{\text {target }}$, for the target probe (in this case, a cantilever with an integrated AFM tip) from Eq. (13). This cantilever of the same width and reflective (gold) coating with an integrated tip is then calibrated using the wedge method for comparison. Henceforth, we will refer to $\alpha_{\text {target }}$ as the lateral force calibration factor for the target probe obtained via the test probe method, and $\alpha_{\text {wedge }}$ is obtained via the wedge method.

\section{B. Comparison of test probe and wedge calibration methods}

To compare $\alpha_{\text {wedge }}$ with $\alpha_{\text {target }}$, we first calibrated the target probe with the wedge method. Next, we calculated the lateral force constant for the target cantilever, using the torsional Sader method and Eq. (17). We then combined Eqs. (13) and (15) to obtain $\alpha_{\text {target }}$ via our test probe method. From this we obtain

$$
\begin{aligned}
\alpha_{\text {target }} & =\frac{k_{\text {lat }}^{\prime}}{s_{\text {lat,target }}} \\
& =\frac{\left(\kappa_{\text {target }} / h_{\text {target }}^{2}\right)\left(L_{\text {target }} / L_{\text {target }}^{\prime}\right)}{s_{\text {lat,test }}\left(T_{\text {target }} / T_{\text {test }}\right)\left(h_{\text {test }} / h_{\text {target }}\right)\left(1+\varepsilon_{\text {test }} / 1+\varepsilon_{\text {target }}\right)} \\
& =\frac{\kappa_{\text {target }} T_{\text {test }} L_{\text {target }}}{s_{\text {lat,test }} h_{\text {test }} h_{\text {target }} T_{\text {target }} L_{\text {target }}^{\prime}}\left(\frac{1+\varepsilon_{\text {target }}}{1+\varepsilon_{\text {test }}}\right),
\end{aligned}
$$

where $L_{\text {target }}$ is the full length of the target cantilever, $L_{\text {target }}^{\prime}$ is the distance from the fixed end of the cantilever to where the tip is attached, and $T_{\text {test }}$ and $T_{\text {target }}$ are the total signals for the colloidal test and target cantilevers, respectively. The ratio $T_{\text {target }} / T_{\text {test }}$ accounts for the different total signal from the test cantilever versus the target cantilever.

The cantilever length, $L_{\text {target }}$, and width, $w_{\text {target }}$, were measured optically, $h_{\text {target }}$ (tip height plus one half of the cantilever thickness) was measured by transmission electron microscopy (TEM), and $h_{\text {test }}$ (colloid radius plus one half of the cantilever thickness) was measured by SEM. ${ }^{27}$ The values are given in Table II. For a colloidal target probe, $h_{\text {target }}$ would be $R(1+\cos \varphi)+t / 2$, where $R$ is the sphere radius. From the Sader method, we calculated the torsional and lateral spring constants for the target cantilever, $\kappa_{\text {target }}$ $=2.91 \pm 0.19 \mathrm{nN} \mathrm{m}$ and $k_{\text {lat,target }}^{\prime}=6.00 \pm 0.41 \mathrm{~N} / \mathrm{m}$, respectively. $T_{\text {target }}$ and $T_{\text {test }}$ were approximately equal for the two cantilevers $(\sim 6 \mathrm{~V})$, which had the same width and coating material. (All parameters and calculated values are provided in Table II.) The measurement uncertainty associated with the test probe method is $6.8 \%$ in the case that $\varepsilon_{\text {target }}=\varepsilon_{\text {test }}$ (see the Appendix). If we ignore the lateral in-plane bending (i.e., neglect $\varepsilon$ or assume that $\varepsilon_{\text {target }}=\varepsilon_{\text {test }}$ ), the test probe lateral calibration factor is $\alpha_{\text {target }}=1.72 \pm 0.12 \mathrm{nN} / \mathrm{mV}$, where we include the standard error and account for the rate and range dependence of the piezoelectric actuator response. If we include the in-plane bending term in Eq. (18), the uncertainty is $7.5 \%$, and $\alpha_{\text {target }}$ becomes $2.39 \pm 0.18 \mathrm{nN} / \mathrm{mV}$, where we have used the values $\varepsilon_{\text {target }}=0.40$ and $\varepsilon_{\text {test }}=0.01$, which are specific to the cantilevers used in this experiment $\left(t_{\text {target }} \sim 3 \mu \mathrm{m}\right.$ by TEM $)$.

For the wedge method, the lateral calibration factor, $\alpha_{\text {wedge }}$, is obtained from Eq. (14) if the normal force calibra- 
tion factor, $\beta$, is known. This is in contrast to the test probe method which yields a lateral calibration factor that is independent of $\beta$. For comparison with the test probe method, $S$ was obtained using the UHV350 AFM for a titanium nitridecoated silicon cantilever with an integrated tip (NT-MDT, Moscow, Russia). Like the test probe measurement, the laser spot was centered on the PSD. We used the silicon "TGG01" wedge-shaped calibration grating (MikroMasch, Wilsonville, Oregon) as the sample, which is described in Varenberg et $a^{25}{ }^{25}$

To complete the lateral force calibration with the wedge method, one must calculate $\beta$ according to Eqs. (3) and (5). The flexural Sader method yields a normal spring constant for this same target cantilever of $k_{\text {norm,target }}^{\prime}$ $=0.074 \pm 0.002 \mathrm{~N} / \mathrm{m}$. Dividing by the normal deflection sensitivity and the cosine of the angle $\varphi=22.5^{\circ}$ between the cantilever's long axis and the sample surface in our instrument, and accounting for the offset of the tip from the end of the cantilever [Eqs. (3) and (5)] yield the normal force calibration factor, $\beta=68.5 \pm 1.9 \mathrm{nN} / \mathrm{V}$. The calculated measurement uncertainty in $\alpha_{\text {wedge }}$ is $4.2 \%$, as shown in the Appendix. The measured wedge lateral calibration factor $\alpha_{\text {wedge }}$ $=2.25 \pm 0.09 \mathrm{nN} / \mathrm{mV}$. The test probe result that accounts for lateral in-plane bending agrees very well with this wedge result, to within $5 \%$ (and they agree within the estimated uncertainties).

\section{Effect of laser spot position on deflection sensitivity and lateral force calibration}

We have observed that the lateral force sensitivity depends strongly on the lateral offset of the PSD with respect to the laser spot position. We studied this dependence and its effect on measurement uncertainty quantitatively by using two methods for the sake of comparison, and to take into account the effect of piezoelectric actuator rate and range effects. In the first method, we translated the PSD laterally from one extreme position to the other and back, obtaining lateral force versus displacement plots with small lateral displacement scans at each PSD position. In the second method, the PSD remained fixed, and we obtained lateral force plots with a much larger lateral scan range and, therefore, a larger deflection range. One example from the first method is shown in Fig. 2. The lateral displacement range was $300 \mathrm{~nm}$. Figure 3 shows both the approach and retract portions of a representative lateral force versus displacement plot taken at the same scan rate as the first method, but with a lateral displacement range of $2 \mu \mathrm{m}$. In this second method, the PSD was positioned with a lateral offset of $\sim 250 \mu \mathrm{m}(\sim 19 \%$ of the width of a PSD sector) so that the laser spot from the untwisted cantilever was displaced to the right-hand side of the PSD, producing a signal of $-5 \mathrm{~V}$ (out of a total of $6 \mathrm{~V}$, thus $\sim 83 \%$ of the spot is incident on the right-hand sectors neglecting truncation of the beam by the finite size of the PSD sectors). The center of the hysteresis loop nearly coincides with the laser passing over the center of the PSD. The hysteresis is due to the dependence of the piezo tube scanners on the applied voltage range. The lateral deflection sensitivity was calculated by taking the derivative of the smoothed force plots.

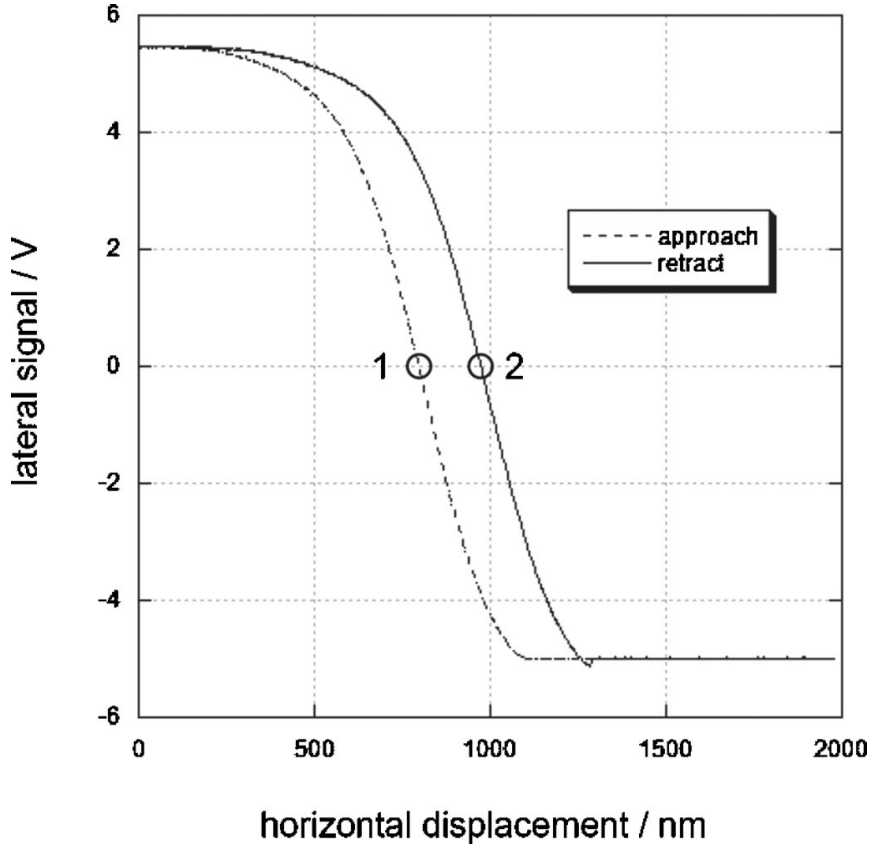

FIG. 3. Lateral force vs. displacement plots. Approach (dashed) and retract (solid) plots taken for durations of $250 \mathrm{~ms}$ in each direction are shown for a displacement range of $2 \mu \mathrm{m}$. The PSD offset $=-5 \mathrm{~V}$ so that the middle of the hysteresis loop coincides with the center of the PSD. The slopes of these data are plotted in Fig. 4, and the points 1 and 2 are included for reference in Fig. 4.

In Fig. 4, the results for both sets of measurements are plotted versus laser spot position in both PSD voltage and spatial displacement. Open and closed symbols refer to the approach and retract data, respectively. Circles indicate PSD offset data (first method), while the triangles correspond to the large scan data (second method). The deflection sensitivities are corrected for the known dependence of the response of our piezo scanners on scan range and scan rate, an effect which is described in detail by Hues et al. ${ }^{28}$ Figure 4 clearly demonstrates by both methods a strong dependence of the lateral deflection sensitivity, $s_{\text {lat }}$, on the position of the laser spot relative to the center of the PSD. We see that $s_{\text {lat }}$ decreases nearly quadratically with increasing offset, a result of the Gaussian distribution of the laser spot.

In Fig. 5, the lateral calibration factor, $\alpha$, is plotted versus PSD lateral laser spot position for the two methods. The laser spot position varies due to lateral PSD offset (method one, circles) or change in cantilever twist angle (larger scan sizes, method two, trieangles). For the larger scan sizes, the unloaded (untwisted) position of the cantilever corresponds to a lateral signal of $-5 \mathrm{~V}$, for a range of $\pm 10 \mathrm{~V}$ (or a displacement range of the colloidal sphere of $\sim 8-10 \mu \mathrm{m})$. The torsional spring constant, $\kappa=2.91 \times 10^{-9} \mathrm{~N} \mathrm{~m}$, was determined by the torsional Sader method. ${ }^{26}$ Closed symbols are corrected for the piezo's rate and range dependence but not for the effect of lateral in-plane bending. Open circles and triangles are test probe data corrected for both piezo and in-plane bending effects, according to Eq. (18). We also determined the lateral force calibration factor's dependence on lateral laser spot offset for the wedge method (hatched squares in Fig. 5, corrected for piezo effects). The plot clearly demonstrates the dependence of both $\alpha_{\text {target }}$ (methods 


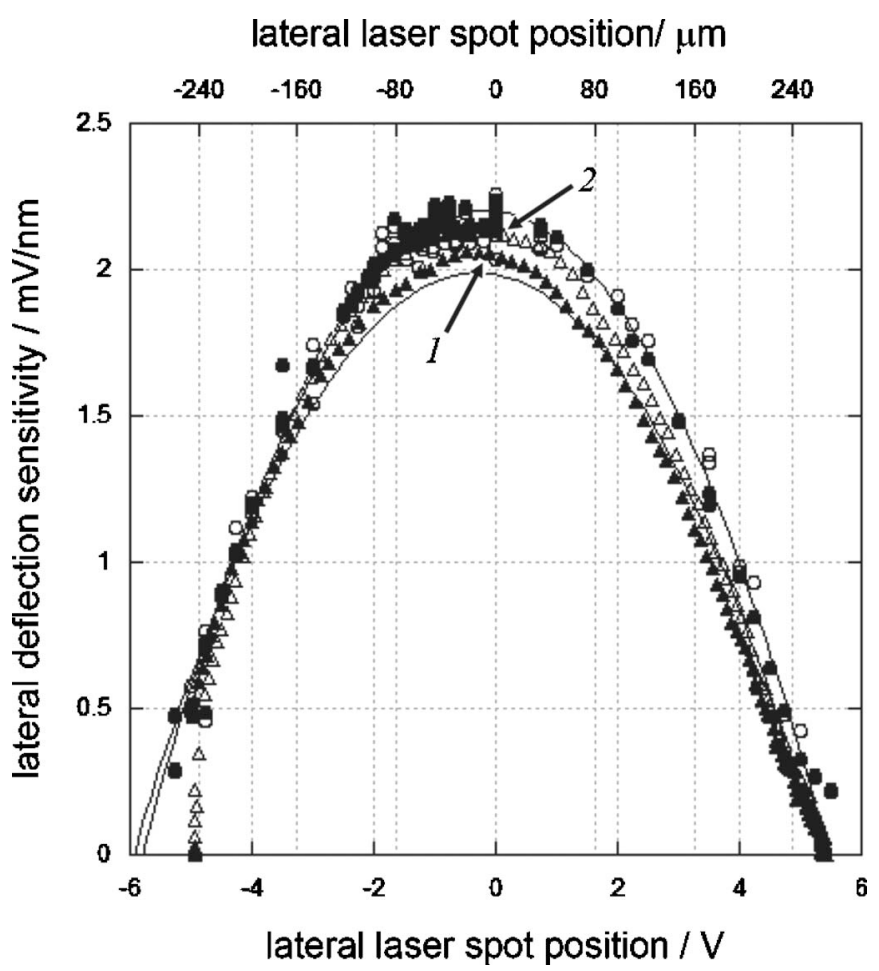

FIG. 4. Lateral deflection sensitivity vs. lateral laser spot position for small (circles) and large (triangles) scan sizes. For the unloaded (untwisted) position, the lateral signal is $-5 \mathrm{~V}$ (for a $\pm 10 \mathrm{~V}$ range), corresponding to $83 \%$ of the total laser intensity incident on the right-hand PSD sectors. The upper abscissa indicates the approximate spatial displacement of the PSD ( $\sim 250 \mu \mathrm{m}$ for the unloaded position) relative to the laser spot position. Open and closed data correspond to approach and retract force plots, respectively. The total signal is approximately constant for the range of values shown. Error bars corresponding to the measurement uncertainty are smaller than the data points. The parabolic fits (to the retract data) indicate good agreement with behavior predicted by a Gaussian distribution. For reference, points 1 and 2 correspond to the slopes of the circled points 1 and 2 on the approach and retract plots in Fig. 3 .

one and two) and $\alpha_{\text {wedge }}$ on the position, $x_{C}$, of the laser spot relative to the center of the PSD.

The lateral deflection sensitivity decreases and the lateral calibration factor increases with the increased lateral offset of the laser spot. With uncertainties in $\alpha_{\text {target }}$ and $\alpha_{\text {wedge }}$ of $6.8 \%$ and $4.2 \%$, respectively, at all offsets for our instrument, the absolute uncertainty in $\alpha$ grows as the PSD offset increases. Similar behavior is observed for the normal deflection sensitivity versus vertical laser spot offset. Thus, our conclusions regarding the lateral sensitivity and calibration apply equally to normal forces.

\section{DISCUSSION}

The test probe method appears to be a reliable means of performing lateral force calibration for AFM. If in-plane bending of the target probe is ignored, the results for the test probe and wedge methods differ by $\sim 25 \%$ (where we have considered the effect of cantilever tilt on the normal force calibration in the wedge method). When we consider the in-plane bending, the values for the test probe and wedge method differ by only $5 \%$, and their error bars overlap. In

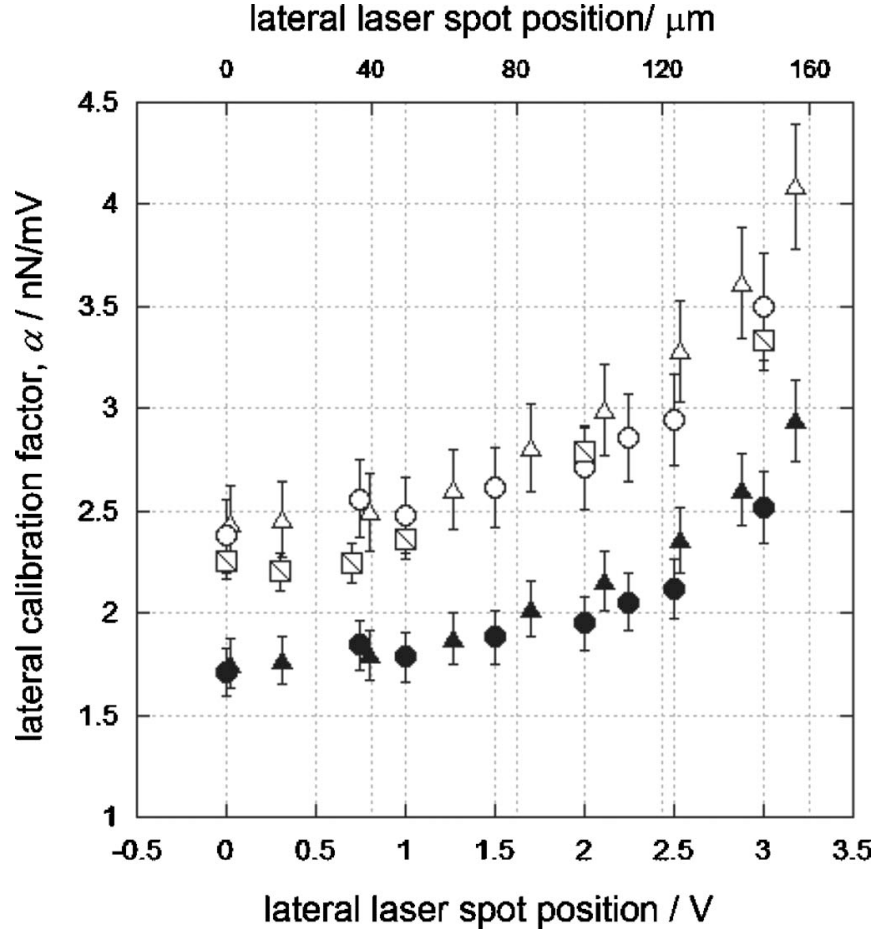

FIG. 5. Lateral calibration factor, $\alpha$, vs. laser spot position, $x_{C}$, relative to the center of the photodiode, showing a clear dependence of lateral calibration factor on $x_{C}$. Closed circles and open triangles are test probe data (with $6.8 \%$ error bars) calculated from the deflection sensitivity for small and large scan sizes, respectively, without accounting for lateral in-plane bending of either the test or target cantilever. Open circles and open triangles are test probe results accounting for in-plane bending (7.5\% error bars). Hatched square data points are the wedge calibration results with the normal force calibration correction for cantilever angle with respect to the sample surface (4.2\% error bars). All data are corrected for the effect of rate and range on piezo response. Agreement is found between the test probe and wedge methods only if lateral in-plane bending is included.

principle, any calibration technique that calculates separately the spring constant and deflection sensitivity, such as the static friction method (for colloidal probes), will remain valid only if lateral in-plane bending is accounted for (or is negligible). The wedge method works around this limitation by direct measurement of lateral and normal force calibration factors, thereby avoiding separate measurement of the lateral spring constant and deflection sensitivity.

The test probe method is similar to Ecke's vertical lever method. However, the challenging step of performing force plots against a calibrated cantilever is no longer necessary. By eliminating the second step in Ecke's method, which limits the calibration to cantilevers with colloidal spheres, it is possible to calibrate cantilevers with any type of tip by using the deflection sensitivity measured from the colloidal test probe's lateral force versus displacement plots as described above.

The precision of any method depends on the uncertainty in each measured parameter used to calculate the calibration factor, leaving room for improvement, e.g., if the cantilever and tip or colloidal sphere dimensions can be measured more precisely. Clearly, the accuracy in force calibration suffers substantially whenever the manufacturer values for cantilever dimensions are used in lieu of direct measurement. Based on the calculations presented in the Appendix, the most sig- 
nificant contribution to uncertainty in $\alpha_{\text {target }}$ arises from the parameter, $h_{\text {target }}$, and due to the strong dependence on the width of the cantilever $\left(\alpha_{\text {target }} \propto w^{4}\right)$. Though $w$ is also a significant source of error in the wedge method, $\alpha_{\text {wedge }}$ depends much more weakly on $w\left(\alpha_{\text {wedge }} \propto w^{2}\right)$. Efforts to improve the precision of the width measurement enhance the accuracy of the calibration. Note that another error source will arise if the cantilever deviates from a perfectly rectangular shape.

We also found that the absolute uncertainty increases significantly versus laser spot position relative to the center of the PSD, and it is therefore important to center the laser spot on the PSD during an experiment and related calibration. In summary, to optimize the precision in force calibration it is important to center the laser spot on the photodiode both vertically and horizontally, and also to account for piezo calibration errors which depend on the scan range and scan rate.

While the uncertainty associated with the wedge method is less than the uncertainty in the new test probe lateral force calibration method presented here, in general, the choice of lateral calibration method is not only a question of accuracy, but it depends on the limitations imposed by the experiment. The test probe method should be used when it is important to avoid contact between the probe(s) in the experiment and any surface other than the sample(s) under investigation. This is crucial in cases where the integrated tip or colloidal sphere has a particular coating that must be preserved. The test probe method may also be used to calculate the lateral force calibration factor at any time for any cantilever of width and reflective coating similar to the test probe. After an experiment is completed and dismantled, it remains possible to calibrate forces with the test probe method with minimal uncertainty.

\section{ACKNOWLEDGMENTS}

The authors are grateful for financial support from the National Science Foundation and from the Army Research Office. In addition, R.J.C. wishes to thank Emre Tepedelenlioğlu and Erin Flater for helpful discussions and comments on this work.

\section{APPENDIX}

From Eq. (18) for the lateral calibration factor calculated with the test probe method, we have

$$
\alpha_{\text {target }}=\frac{\kappa T_{\text {test }} L_{\text {target }}}{s_{\text {lat, test }} h_{\text {test }} h_{\text {target }} T_{\text {target }} L_{\text {target }}^{\prime}} .
$$

According to the torsional Sader method, the torsional spring constant, $\kappa$, is given by

$$
\kappa=0.1592 \rho w^{4} L Q_{\text {tors }} \omega_{\text {tors }}^{2} \Gamma_{i, \text { tors }}\left(\omega_{\text {tors }}\right),
$$

where $\rho$ is the fluid density of the environment $\left(1.18 \mathrm{~kg} / \mathrm{m}^{3}\right.$ for air), $Q_{\text {tors }}$ and $\omega_{\text {tors }}$ are the quality factor and frequency of the fundamental torsional resonance, respectively, and $\Gamma_{\mathrm{i}, \text { tors }}\left(\omega_{\text {tors }}\right)$ is the imaginary part of the torsional hydrodynamic function, which can be obtained from a table or a numeric function.

Using standard error analysis and denoting the uncertainty in any physical quantity $P$ as $\delta P{ }^{29}$ The uncertainty in the test probe lateral force calibration factor, $\delta \alpha_{\text {test }}$, is given by

$$
\begin{aligned}
\delta \alpha_{\text {target }}= & \alpha_{\text {target }}\left[\left(\frac{4 \delta w_{\text {target }}}{w_{\text {target }}}\right)^{2}+\left(\frac{\delta Q_{\text {tors }}}{Q_{\text {tors }}}\right)^{2}+\left(\frac{2 \delta f_{\text {tors }}}{f_{\text {tors }}}\right)^{2}\right. \\
& +\left(\frac{\delta \Gamma_{i, \text { tors }}}{\Gamma_{i, \text { tors }}}\right)^{2}+\left(\frac{2 \delta L_{\text {target }}}{L_{\text {target }}}\right)^{2}+\left(\frac{\delta L_{\text {target }}^{\prime}}{L_{\text {target }}^{\prime}}\right)^{2} \\
& +\left(\frac{\delta h_{\text {test }}}{h_{\text {test }}}\right)^{2}+\left(\frac{\delta h_{\text {target }}}{h_{\text {target }}}\right)^{2}+\left(\frac{\delta s_{\text {lat,test }}}{s_{\text {lat,test }}}\right)^{2} \\
& \left.+\left(\frac{\delta T_{\text {test }}}{T_{\text {test }}}\right)^{2}+\left(\frac{\delta T_{\text {target }}}{T_{\text {target }}}\right)^{2}\right]^{1 / 2},
\end{aligned}
$$

where

$$
\left(\frac{\delta s_{\text {lat,test }}}{s_{\text {lat,test }}}\right)^{2}=\left(\frac{\delta V_{\text {lat }}}{V_{\text {lat }}}\right)^{2}+\left(\frac{\delta V_{\text {lat,piezo }}}{V_{\text {lat,piezo }}}\right)^{2} .
$$

$V_{\text {lat }}$ and $V_{\text {lat,piezo }}$ are the lateral PSD signal and voltage applied to the lateral piezo, respectively.

The lateral force calibration factor from the wedge calibration method is given by

$$
\alpha_{\text {wedge }}=S \times \beta .
$$

Since $S$ and $\beta$ are independent measurements, the uncertainty, $\delta \alpha_{\text {wedge }}$, may be written

$$
\delta \alpha_{\text {wedge }}=\alpha_{\text {wedge }}\left[\left(\frac{\delta S}{S}\right)^{2}+\left(\frac{\delta \beta}{\beta}\right)^{2}\right]^{1 / 2}
$$

where

$$
\left(\frac{\delta \beta}{\beta}\right)^{2}=\left(\frac{\delta k_{\text {norm }}^{\prime}}{k_{\text {norm }}^{\prime}}\right)^{2}+\left(\frac{\delta s_{\text {norm }}}{s_{\text {norm }}}\right)^{2} .
$$

Using the flexural Sader method,

$$
k_{\text {norm }}=0.1906 \rho w^{2} L Q_{\text {norm }} \omega_{\text {norm }}^{2} \Gamma_{i, \text { norm }}\left(\omega_{\text {norm }}\right),
$$

and (A6) becomes

$$
\begin{aligned}
\delta \alpha_{\text {wedge }}= & \alpha_{\text {wedge }}\left[\left(\frac{\delta S}{S}\right)^{2}+\left(\frac{2 \delta w}{w}\right)^{2}+\left(\frac{4 \delta L}{L}\right)^{2}\right. \\
& +\left(\frac{3 \delta L^{\prime}}{L^{\prime}}\right)^{2}+\left(\frac{\delta Q_{\text {norm }}}{Q_{\text {norm }}}\right)^{2}+\left(\frac{2 \delta f_{\text {norm }}}{f_{\text {norm }}}\right)^{2} \\
& \left.+\left(\frac{\delta \Gamma_{i, \text { norm }}}{\Gamma_{i, \text { norm }}}\right)^{2}+\left(\frac{\delta s_{\text {norm }}}{s_{\text {norm }}}\right)^{2}\right]^{1 / 2} .
\end{aligned}
$$

Note that the wedge calibration uses one fewer parameter (no dependence on $h$ ) than the test probe method, and it depends much more weakly on $w$, which is a large source of error in both methods. While $w$ is the most significant source of error 
in the test probe method, $L$ is equally significant in the wedge method.

In the wedge method, the sensitivity factor, $S$, depends on the slopes, $u_{1}$ and $u_{2}$, of the lateral versus normal signal plots for the two inclined planes; and $S$ depends on the difference, $d_{12}$, between the slopes of the friction loop offset versus normal signal plots. $S$ is written in terms of the angles $\theta_{1}$ and $\theta_{2}$, and the slopes $u_{1}, u_{2}$, and $d_{12}$ in the following way:

$$
\begin{aligned}
& S\left(u_{1}, u_{2}, d_{12}\right) \\
& \quad=\frac{\left(u_{1} / u_{2}\right) \sin \left(2 \theta_{1}\right)-\sin \left(2 \theta_{2}\right)}{d_{12} \cos \left(2 \theta_{2}\right)+\sqrt{d_{12}^{2}-\left[u_{1} \sin \left(2 \theta_{1}\right)-u_{2} \sin \left(2 \theta_{2}\right)\right]^{2}}} .
\end{aligned}
$$

Using the standard method of calculating error propagation, we calculate the partial derivatives of $S$ with respect to each independent variable: ${ }^{29}$

$$
\begin{aligned}
\frac{\partial S\left(u_{1}, u_{2}, d_{12}\right)}{\partial u_{1}}= & \frac{\left(1 / u_{2}\right) \sin \left(2 \theta_{1}\right)}{d_{12} \cos \left(2 \theta_{2}\right)+\sqrt{d_{12}^{2}-\left[u_{1} \sin \left(2 \theta_{1}\right)-u_{2} \sin \left(2 \theta_{2}\right)\right]^{2}}} \\
& +\frac{\left[\left(u_{1} / u_{2}\right) \sin \left(2 \theta_{1}\right)-\sin \left(2 \theta_{2}\right)\right]\left[u_{1} \sin \left(2 \theta_{1}\right)-u_{2} \sin \left(2 \theta_{2}\right)\right] \sin \left(2 \theta_{1}\right)}{\left\{d_{12} \cos \left(2 \theta_{2}\right)+\sqrt{d_{12}^{2}-\left[u_{1} \sin \left(2 \theta_{1}\right)-u_{2} \sin \left(2 \theta_{2}\right)\right]^{2}}\right\}^{2} V \sqrt{d_{12}^{2}-\left[u_{1} \sin \left(2 \theta_{1}\right)-u_{2} \sin \left(2 \theta_{2}\right)\right]^{2}}}, \\
\frac{\partial S\left(u_{1}, u_{2}, d_{12}\right)}{\partial u_{2}}= & \frac{\left(-u_{1} / u_{2}^{2}\right) \sin \left(2 \theta_{1}\right)}{d_{12} \cos \left(2 \theta_{2}\right)+\sqrt{d_{12}^{2}-\left[u_{1} \sin \left(2 \theta_{1}\right)-u_{2} \sin \left(2 \theta_{2}\right)\right]^{2}}} \\
& +\frac{-\left[\left(u_{1} / u_{2}\right) \sin \left(2 \theta_{1}\right)-\sin \left(2 \theta_{2}\right)\right]\left[u_{1} \sin \left(2 \theta_{1}\right)-u_{2} \sin \left(2 \theta_{2}\right)\right] \sin \left(2 \theta_{2}\right)}{\left\{d_{12} \cos \left(2 \theta_{2}\right)+\sqrt{d_{12}^{2}-\left[u_{1} \sin \left(2 \theta_{1}\right)-u_{2} \sin \left(2 \theta_{2}\right)\right]^{2}}\right\}^{2} \sqrt{d_{12}^{2}-\left[u_{1} \sin \left(2 \theta_{1}\right)-u_{2} \sin \left(2 \theta_{2}\right)\right]^{2}}}, \\
\frac{\partial S\left(u_{1}, u_{2}, d_{12}\right)}{\partial d_{12}}= & \frac{-\left[\left(u_{1} / u_{2}\right) \sin \left(2 \theta_{1}\right)-\sin \left(2 \theta_{2}\right)\right]}{\left\{d_{12} \cos \left(2 \theta_{2}\right)+\sqrt{d_{12}^{2}-\left[u_{1} \sin \left(2 \theta_{1}\right)-u_{2} \sin \left(2 \theta_{2}\right)\right]^{2}}\right\}^{2}}\left\{\cos \left(2 \theta_{2}\right)+\frac{d_{12}}{\sqrt{d_{12}^{2}-\left[u_{1} \sin \left(2 \theta_{1}\right)-u_{2} \sin \left(2 \theta_{2}\right)\right]^{2}}}\right\} .
\end{aligned}
$$

The uncertainty $\delta S$ is given by

$$
(\delta S)^{2}=\left(\frac{\partial S}{\partial u_{1}} \delta u_{1}\right)^{2}+\left(\frac{\partial S}{\partial u_{2}} \delta u_{2}\right)^{2}+2\left(\frac{\partial S}{\partial d_{12}} \delta d_{12}\right)^{2}
$$

where the factor of 2 in the last term arises because $d_{12}$ is a difference between the slopes of two offset versus load plots. In general, $\delta S$ will depend on the friction coefficient, $\mu$, of the calibration grating and will increase with increasing $\mu$ at a rate that depends on the uncertainty in the measured width

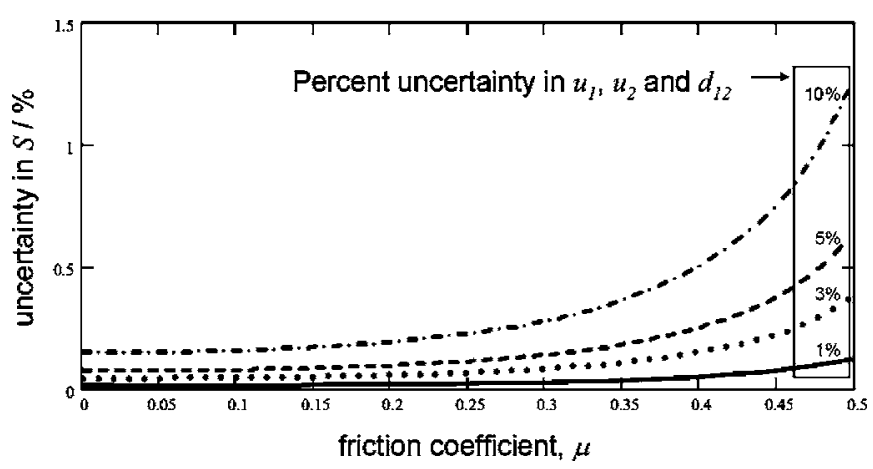

FIG. 6. Percent error in the wedge method sensitivity factor, $S$, vs. friction coefficient, $\mu$ (assuming the two sloped surfaces have the same $\mu$ ). Solid, dotted, dashed, and dash-dotted lines correspond to $1 \%, 3 \%, 5 \%$, and $10 \%$ uncertainties, respectively, in the measured friction loop half-widths, $u_{1}$ and $u_{2}$, and friction loop offset, $d_{12}$. The error in $S$ becomes unreasonable at high friction coefficients and large uncertainties in these measured values. and offset slopes. Figure 6 shows the dependence of the percent uncertainty in $S(\delta S / S)$ on $\mu, u_{1}, u_{2}$, and $d_{12}$. As $\mu$ increases, the percent uncertainty in $u_{1}, u_{2}$, and $d_{12}$ becomes increasingly important to the calibration, and the percent uncertainty in $S$ grows to unreasonable large values.

In the current experiment, both the friction coefficient and the percent uncertainties are small enough that $\delta S$ remains reasonable at $\sim 3 \%$. Table II lists the percent uncertainties in each of the measured and calculated parameters for both calibration methods based on the equations given above.

${ }^{1}$ R. W. Carpick and M. Salmeron, Chem. Rev. (Washington, D.C.) 97, 1163 (1997).

${ }^{2}$ E. Gnecco, R. Bennewitz, A. Socoliuc, and E. Meyer, Wear 254, 859 (2003)

${ }^{3}$ G. Meyer and N. M. Amer, Appl. Phys. Lett. 53, 1045 (1988).

${ }^{4}$ G. Meyer and N. M. Amer, Appl. Phys. Lett. 53, 2400 (1988).

${ }^{5}$ G. Meyer and N. M. Amer, Appl. Phys. Lett. 57, 2089 (1990).

${ }^{6}$ R. J. Cannara, M. J. Brukman, and R. W. Carpick, Rev. Sci. Instrum. 76, 053706 (2005).

${ }^{7}$ J. E. Sader, Rev. Sci. Instrum. 70, 3967 (1999); http:// www.ampc.ms.unimelb.edu.au/afm/calibration.html\#normal

${ }^{8}$ In J. E. Sader's papers, the normal spring constant for the full length of the cantilever is referred to as $k_{\text {flex }}$, which we call $k_{\text {norm }}$ for clarity here.

${ }^{9}$ While spring constant calibration may be performed at different air pressures and for arbitrary geometries using Sader's new methodology, all spring constant calibrations for this work were performed at 760 Torr using rectangular cantilevers. [J. E. Sader, J. Pacifico, C. P. Green, and P. Mulvaney, J. Appl. Phys. 97, 124903 (2005).]

${ }^{10}$ C. A. Clifford and M. P. Seah, Nanotechnology 16, 1666 (2005). 
${ }^{11}$ N. Morel, M. Ramonda, and P. Tordjeman, Appl. Phys. Lett. 86, 163103 (2005).

${ }^{12}$ C. T. Gibson, D. A. Smith, and C. J. Roberts, Nanotechnology 16, 234 (2005).

${ }^{13}$ F. P. Beer, E. R. Johnston, Jr., and J. T. Dewolf, Mechanics of Materials, 3rd ed. (McGraw-Hill, New York, 2001), p. 762.

${ }^{14}$ This is strictly only true for materials with positive Poisson ratios. For example, for linear isotropic materials, $G=E / 2(1+v)$; therefore, $G<E$ if $v>0$. Certain materials with negative Poisson ratios exist, and, in some of these special cases, when $v<-0.5, G$ can be larger than $E$. [R. S. Lakes, Science 235, 1038 (1987); Adv. Mater. (Weinheim, Ger.) 5, 293 (1993)]. ${ }^{15}$ R. W. Carpick, D. F. Ogletree, and M. Salmeron, Appl. Phys. Lett. 70, 1548 (1997).

${ }^{16}$ M. A. Lantz, S. J. O'shea, A. C. F. Hoole, and M. E. Welland, Appl. Phys. Lett. 70, 970 (1997)

${ }^{17}$ R. G. Cain, S. Biggs, and N. W. Page, J. Colloid Interface Sci. 227, 55 (2000).

${ }^{18}$ J. E. Sader, and C. P. Green, Rev. Sci. Instrum. 75, 878 (2004).

${ }^{19}$ E. Liu, B. Blanpain, and J. P. Celis, Wear 192, 141 (1996).

${ }^{20}$ S. Ecke, R. Raiteri, E. Bonaccurso, C. Reiner, H.-J. Deiseroth, and H.-J. Butt, Rev. Sci. Instrum. 72, 4164 (2001).

${ }^{21}$ J. P. Cleveland, S. Manne, D. Bocek, and P. K. Hansma, Rev. Sci. Instrum.
64, 403 (1993).

${ }^{22}$ J. Stiernstedt, M. W. Rutland, and P. Attard, Rev. Sci. Instrum. 76, 262508 (2005).

${ }^{23}$ D. Choi, Ph.D. thesis, POSTECH University, 2006.

${ }^{24}$ D. F. Ogletree, R. W. Carpick, and M. Salmeron, Rev. Sci. Instrum. 67, 3298 (1996).

${ }^{25}$ M. Varenberg, I. Etsion, and G. Halperin, Rev. Sci. Instrum. 74, 3362 (2003).

${ }^{26}$ C. P. Green, H. Lioe, J. P. Cleveland, R. Proksch, P. Mulvaney, and J. E. Sader, Rev. Sci. Instrum. 75, 1988 (2004); http:// www.ampc.ms.unimelb.edu.au/afm/calibration.html\#torsional.

${ }^{27}$ In general, we use TEM to measure probe geometries and tip structure, and SEM is used for cases where the cantilevers charge up or experience excess drift in the TEM. Optical microscopy can be used in many cases, e.g., for colloidal spheres, although the accuracy and precision will be less.

${ }^{28}$ S. M. Hues, C. F. Draper, K. Lee, and R. J. Colton, Rev. Sci. Instrum. 65, 1561 (1994).

${ }^{29}$ L. Lyons, A Practical Guide to Data Analysis for Physical Science Students (Cambridge University Press, New York, 1991), pp. 25-6.

${ }^{30}$ R. G. Cain, M. G. Reitsma, S. Biggs, and N. W. Page, Rev. Sci. Instrum. 72, 3304 (2001) 